\title{
On almost and weakly $\delta$-semicontinuous multifunctions
}

\author{
Erdal Ekici
}




\title{
ON ALMOST AND WEAKLY $\delta$-SEMICONTINUOUS MULTIFUNCTIONS
}

\author{
ERDAL EKICI
}

Received 4 February, 2005

\begin{abstract}
The aim of this paper is to introduce two new classes of multifunctions called almost $\delta$-semicontinuous multifunctions and weakly $\delta$-semicontinuous multifunctions which are generalization of $\delta$-semicontinuous multifunctions. In this paper we obtain several properties of such multifunctions.
\end{abstract}

2000 Mathematics Subject Classification: 54C08, 54C60

Keywords: $\delta$-semicontinuous, almost continuity, weakly continuity, multifunction

\section{INTRODUCTION}

Weaker and stronger forms of open sets play an important role in topologically spaces. By using these sets many authors introduced and studied various types of generalizations of continuity. In this paper, we introduce the notions of almost and weakly $\delta$-semicontinuous multifunctions and investigate the relationships among $\delta$ semicontinuity, almost $\delta$-semicontinuity and weakly $\delta$-semicontinuity for multifunctions.

In this paper, the spaces $X$ and $Y$ mean topological spaces on which no separation axioms are assumed unless explicitly stated. For a subset $A$ of $X, \operatorname{cl}(A)$ and $\operatorname{int}(A)$ represent the closure of $A$ and the interior of $A$, respectively.

A subset $A$ of a space $X$ is said to be regular open if $A=\operatorname{int}(\operatorname{cl}(A))$ [15]. The complement of a regular open set is said to be regular closed. The $\delta$-interior [16] of a subset $A$ of $(X, \tau)$ is the union of all regular open sets of $X$ contained in $A$ is denoted by $\delta$-int $(A)$. A subset $A$ is called $\delta$-open [16] if $A=\delta$-int $(A)$. The complement of $\delta$-open set is called $\delta$-closed. The $\delta$-closure of a subset $A$ is defined by $\{x \in X$ : $A \cap \operatorname{int}(\operatorname{cl}(U)) \neq \varnothing, U \in \tau$ and $x \in U\}$ and is denoted by $\delta$-cl $(A)$.

A subset $A$ of a space $X$ is said to be $\delta$-semiopen [10] (resp., semi-open [4], preopen [6], $\beta$-open [1]) if $A \subset \operatorname{cl}(\delta$-int $(A))$ (resp., $A \subset \operatorname{cl}(\operatorname{int}(A)), A \subset \operatorname{int}(\operatorname{cl}(A)), A \subset$ $\operatorname{cl}(\operatorname{int}(\operatorname{cl}(A))))$. The family of all $\delta$-semiopen (resp., semi-open, preopen, $\beta$-open, $\delta$ open, $\delta$-semiclosed) sets of $X$ is denoted by $\delta S O(X)$ (resp., $S O(X), P O(X), \beta O(X)$, $\delta O(X), \delta S C(X))$. The family of all $\delta$-semiopen (resp., semi-open, $\delta$-semiclosed) sets 
of $X$ containing a point $x \in X$ is denoted by $\delta S O(X, x)$ (resp., $S O(X, x), \delta S C(X, x)$ ). The complement of a $\delta$-semiopen set is said to be $\delta$-semiclosed. The intersection of all $\delta$-semiclosed sets (resp., semi-closed) of $X$ containing $A$ is called the $\delta$-semiclosure [10] (resp., semi-closure [2]) of $A$ and is denoted by $\delta$-scl( $A)(\operatorname{resp} ., \operatorname{scl}(A)$ ). The union of all $\delta$-semiopen (resp., semi-open) sets of $X$ contained in $A$ is called $\delta$ semi-interior (resp., semi-interior) of $A$ and is denoted by $\delta$-sint $(A)$ (resp., $\operatorname{sint}(A)$ ).

Definition 1. A multifunction $F: X \rightarrow Y$ is said to be:

(1) upper quasi-continuous [12] if for each $x \in X$ and each open set $V$ of $Y$ containing $F(x)$, there exists $U \in S O(X, x)$ such that $F(U) \subset V$,

(2) lower quasi-continuous [12] if for each $x \in X$ and each open set $V$ of $Y$ such that $F(x) \cap V \neq \varnothing$, there exists $U \in S O(X, x)$ such that $F(u) \cap V \neq \varnothing$ for every $u \in U$.

Definition 2. For a multifunction $F: X \rightarrow Y$, the graph multifunction $G_{F}: X \rightarrow$ $X \times Y$ is defined in the following way: $G_{F}(x)=\{x\} \times F(x)$ for every $x \in X$, and the subset $\{\{x\} \times F(x): x \in X\} \subset X \times Y$ is called the multigraph of $F$ and is denoted by $G(F)[14]$.

Definition 3. A multifunction $F: X \rightarrow Y$ is said to be:

(1) lower $\delta$-semicontinuous [3] if for each $x \in X$ and each open set $V$ such that $x \in F^{-}(V)$, there exists a $\delta$-semiopen set $U$ containing $x$ such that $U \subset$ $F^{-}(V)$,

(2) upper $\delta$-semicontinuous [3] if for each $x \in X$ and each open set $V$ such that $x \in F^{+}(V)$, there exists a $\delta$-semiopen set $U$ containing $x$ such that $U \subset F^{+}(V)$.

Lemma 1 ([7,9]). For a multifunction $F: X \rightarrow Y$, the following assertions hold:

(1) $G_{F}^{+}(A \times B)=A \cap F^{+}(B)$,

(2) $G_{F}^{-}(A \times B)=A \cap F^{-}(B)$ for any subsets $A \subset X$ and $B \subset Y$.

Lemma 2 ([7]). $\operatorname{scl}(V)=\operatorname{int}(\operatorname{cl}(V))$ for every preopen set $V$ of a space $X$.

\section{Almost And WEAKLy $\delta$-SEMiCONTINUOUS MUlTifunctions}

In this section, characterizations of almost and weakly $\delta$-semicontinuous multifunctions and properties of them are introduced.

Definition 4. A multifunction $F: X \rightarrow Y$ is said to be:

(1) lower almost $\delta$-semicontinuous if for each $x \in X$ and each open set $V$ such that $x \in F^{-}(V)$, there exists a $\delta$-semiopen set $U$ containing $x$ such that $U \subset$ $F^{-}(\operatorname{int}(\operatorname{cl}(V)))$,

(2) upper almost $\delta$-semicontinuous if for each $x \in X$ and each open set $V$ such that $x \in F^{+}(V)$, there exists a $\delta$-semiopen set $U$ containing $x$ such that $U \subset F^{+}(\operatorname{int}(\operatorname{cl}(V)))$. 
Theorem 1. Let $F: X \rightarrow Y$ be a multifunction. Then the following statements are equivalent:

(1) $F$ is upper almost $\delta$-semicontinuous multifunction,

(2) $F^{+}(\operatorname{int}(\operatorname{cl}(V))) \in \delta S O(X)$ for any open set $V \subset Y$,

(3) $F^{-}(\operatorname{cl}(\operatorname{int}(K))) \in \delta S C(X)$ for any closed set $K \subset Y$,

(4) $F^{+}(G) \in \delta S O(X)$ for any regular open set $G$ of $Y$,

(5) $F^{-}(K) \in \delta S C(X)$ for any regular closed set $K$ of $Y$,

(6) for each $x \in X$ and each open set $V$ containing $F(x)$, there exists a $\delta$ semiopen set $U$ containing $x$ such that $F(U) \subset \operatorname{scl}(V)$,

(7) $F^{+}(V) \subset \delta-\operatorname{sint}\left(F^{+}(\operatorname{scl}(V))\right)$ for every open set $V$ in $Y$,

(8) $\delta-\operatorname{scl}\left(F^{-}(\operatorname{sint}(K))\right) \subset F^{-}(K)$ for every closed set $K$ of $Y$,

(9) $\delta-\operatorname{scl}\left(F^{-}(\operatorname{cl}(\operatorname{int}(K)))\right) \subset F^{-}(K)$ for every closed set $K$ of $Y$,

(10) $\delta-\operatorname{scl}\left(F^{-}(V)\right) \subset F^{-}(\operatorname{cl}(V))$ for every $V \in \beta O(Y)$,

(11) $\delta$-scl $\left(F^{-}(V)\right) \subset F^{-}(\operatorname{cl}(V))$ for every $V \in S O(Y)$,

(12) $F^{+}(V) \subset \delta-\operatorname{sint}\left(F^{+}(\operatorname{int}(\operatorname{cl}(V)))\right)$ for every $V \in P O(Y)$.

Proof. (1) $\Rightarrow(2)$ : Let $V$ be any open set of $Y$ and $x \in F^{+}(\operatorname{int}(\operatorname{cl}(V)))$. By (1), there exists $U_{x} \in \delta S O(X, x)$ such that $U_{x} \subset F^{+}(\operatorname{int}(\operatorname{cl}(V)))$. Hence, $F^{+}(\operatorname{int}(\operatorname{cl}(V))) \in$ $\delta S O(X)$.

$(2) \Rightarrow(1)$ : Let $V$ be any open set of $Y$ and $x \in F^{+}(V)$. By (2), $F^{+}(\operatorname{int}(\operatorname{cl}(V))) \in$ $\delta S O(X)$. Take $U=F^{+}(\operatorname{int}(\operatorname{cl}(V)))$. Then, $F(U) \subset \operatorname{int}(\operatorname{cl}(V))$. Hence, $F$ is upper almost $\delta$-semicontinuous.

(2) $\Leftrightarrow(3)$ : Let $K$ be any closed set of $Y$. Then, $Y \backslash K$ is an open set of $Y$. By (2), $F^{+}(\operatorname{int}(\operatorname{cl}(Y \backslash K))) \in \delta S O(X)$. Since $\operatorname{int}(\operatorname{cl}(Y \backslash K))=Y \backslash \operatorname{cl}(\operatorname{int}(K))$, it follows that $F^{+}(\operatorname{int}(\operatorname{cl}(Y \backslash K)))=X \backslash F^{-}(\operatorname{cl}(\operatorname{int}(K)))$. We obtain that $F^{-}(\operatorname{cl}(\operatorname{int}(K)))$ is $\delta$-semiclosed in $X$. The converse implication is proved by analogy.

$(2) \Leftrightarrow(4),(3) \Leftrightarrow(5)$ : Obvious.

(1) $\Leftrightarrow(6)$ : Let $x \in X$ and $V$ be an open set containing $F(x)$. By (1) and Lemma 2, there exists a $\delta$-semiopen set $U$ containing $x$ such that $U \subset F^{+}(\operatorname{scl}(V))$. The converse implication is obtained similarly.

(5) $\Rightarrow$ (7): Let $V$ be an open set in $Y$ and $x \in F^{+}(V)$. Then we have $F(x) \subset V \subset$ $\operatorname{scl}(V)$ and hence $x \in F^{+}(\operatorname{scl}(V))=X \backslash F^{-}(Y \backslash \operatorname{scl}(V))$. Since $Y \backslash \operatorname{scl}(V)$ is regular closed, then $F^{-}(Y \backslash \operatorname{scl}(V))$ is $\delta$-semiclosed in $Y$. Therefore, $F^{+}(\operatorname{scl}(V))$ is $\delta$ semiopen and hence $x \in \delta$-sint $\left(F^{+}(\operatorname{scl}(V))\right)$. Consequently, we obtain $F^{+}(V) \subset \delta$ $\operatorname{sint}\left(F^{+}(\operatorname{scl}(V))\right)$.

(7) $\Rightarrow(8)$ : Let $K$ be any closed set of $Y$. Then, since $Y \backslash K$ is open, we obtain $X \backslash F^{-}(K)=F^{+}(Y \backslash K) \subset \delta-\operatorname{sint}\left(F^{+}(\operatorname{scl}(Y \backslash K))\right)=\delta-\operatorname{sint}\left(F^{+}(Y \backslash \operatorname{sint}(K))\right)=$ $\delta-\operatorname{sint}\left(X \backslash F^{-}(\operatorname{sint}(K))\right)=X \backslash \delta-\operatorname{scl}\left(F^{-}(\operatorname{sint}(K))\right)$. We thus obtain the inclusion $\delta-\operatorname{scl}\left(F^{-}(\operatorname{sint}(K))\right) \subset F^{-}(K)$.

$(8) \Rightarrow(9)$ : Since $\operatorname{sint}(K)=\operatorname{cl}(\operatorname{int}(K))$ for every closed set $K$, the proof is obvious.

$(9) \Rightarrow(5)$ : Let $K$ be any regular closed set in $Y$. Then $\delta-\operatorname{scl}\left(F^{-}(\operatorname{cl}(\operatorname{int}(K)))\right)=\delta$ $\operatorname{scl}\left(F^{-}(K)\right) \subset F^{-}(K)$. We obtain that $F$ is upper almost $\delta$-semicontinuous. 
$(5) \Rightarrow(10)$ : Let $V$ be any $\beta$-open set in $Y$. Since $\operatorname{cl}(V)$ is regular closed, $F^{-}(\operatorname{cl}(V))$ is $\delta$-semiclosed and $F^{-}(V) \subset F^{-}(\operatorname{cl}(V))$. Hence, $\delta$ - $\operatorname{scl}\left(F^{-}(V)\right) \subset F^{-}(\operatorname{cl}(V))$.

$(10) \Rightarrow(11)$ : Obvious.

$(11) \Rightarrow(12)$ : Let $V$ be a preopen set. Then we have the inclusions $V \subset \operatorname{int}(\operatorname{cl}(V))$ and $\operatorname{cl}(\operatorname{int}(Y \backslash V)) \subset Y \backslash V$. Moreover, since the set $\operatorname{cl}(\operatorname{int}(Y \backslash V))$ is semi-open, it follows that $X \backslash \delta$-sint $\left(F^{+}(\operatorname{int}(\operatorname{cl}(V)))\right)=\delta-\operatorname{scl}\left(X \backslash F^{+}(\operatorname{int}(\operatorname{cl}(V)))\right)=\delta-\operatorname{scl}\left(F^{-}(Y \backslash\right.$ $\operatorname{int}(\operatorname{cl}(V))))=\delta-\operatorname{scl}\left(F^{-}(\operatorname{cl}(\operatorname{int}(Y \backslash V)))\right) \subset F^{-}(\operatorname{cl}(\operatorname{int}(Y \backslash V))) \subset F^{-}(Y \backslash V) \subset X \backslash$ $F^{+}(V)$. Hence, we obtain $F^{+}(V) \subset \delta$-sint $\left(F^{+}(\operatorname{int}(\operatorname{cl}(V)))\right)$.

$(12) \Rightarrow(4)$ : Let $V$ be a regular open set. Since $V$ is preopen, we get $F^{+}(V) \subset \delta$ $\operatorname{sint}\left(F^{+}(\operatorname{int}(\operatorname{cl}(V)))\right)=\delta$-sint $\left(F^{+}(V)\right)$. Hence, $F^{+}(V)$ is $\delta$-semiopen.

Theorem 2. Let $F: X \rightarrow Y$ be a multifunction. Then the following statements are equivalent:

(1) $F$ is lower almost $\delta$-semicontinuous multifunction,

(2) $F^{-}(\operatorname{int}(\operatorname{cl}(V))) \in \delta S O(X)$ for any open set $V \subset Y$,

(3) $F^{+}(\operatorname{cl}(\operatorname{int}(K))) \in \delta S C(X)$ for any closed set $K \subset Y$,

(4) $F^{-}(G) \in \delta S O(X)$ for any regular open set $G$ of $Y$,

(5) $F^{+}(K) \in \delta S C(X)$ for any regular closed set $K$ of $Y$,

(6) for each $x \in X$ and each open set $V$ such that $F(x) \cap V \neq \varnothing$, there exists a $\delta$-semiopen set $U$ containing $x$ such that $F(u) \cap \operatorname{scl}(V) \neq \varnothing$ for every $u \in U$,

(7) $F^{-}(V) \subset \delta$-sint $\left(F^{-}(\operatorname{scl}(V))\right)$ for every open set $V$ in $Y$,

(8) $\delta$ - $\operatorname{scl}\left(F^{+}(\operatorname{sint}(K))\right) \subset F^{+}(K)$ for every closed set $K$ of $Y$,

(9) $\delta-\operatorname{scl}\left(F^{+}(\operatorname{cl}(\operatorname{int}(K)))\right) \subset F^{+}(K)$ for every closed set $K$ of $Y$,

(10) $\delta-\operatorname{scl}\left(F^{+}(V)\right) \subset F^{+}(\operatorname{cl}(V))$ for every $V \in \beta O(Y)$,

(11) $\delta-\operatorname{scl}\left(F^{+}(V)\right) \subset F^{+}(\operatorname{cl}(V))$ for every $V \in S O(Y)$,

(12) $F^{-}(V) \subset \delta-\operatorname{sint}\left(F^{-}(\operatorname{int}(\operatorname{cl}(V)))\right)$ for every $V \in P O(Y)$.

Proof. It is similar to that of the previous theorem.

Definition 5. A multifunction $F: X \rightarrow Y$ is said to be:

(1) lower weakly $\delta$-semicontinuous if for each $x \in X$ and each open set $V$ of $Y$ such that $x \in F^{-}(V)$, there exists a $\delta$-semiopen set $U$ containing $x$ such that $U \subset F^{-}(\mathrm{cl}(V))$,

(2) upper weakly $\delta$-semicontinuous if for each $x \in X$ and each open set $V$ of $Y$ such that $x \in F^{+}(V)$, there exists a $\delta$-semiopen set $U$ containing $x$ such that $U \subset F^{+}(\operatorname{cl}(V))$.

Theorem 3. Let $F: X \rightarrow Y$ be a multifunction. Then the following statements are equivalent:

(1) $F$ is upper weakly $\delta$-semicontinuous multifunction,

(2) for each $x \in X$ and each open set $V$ containing $F(x)$, there exists a $\delta$ semiopen set $U$ containing $x$ such that $F(U) \subset \operatorname{cl}(V)$, 
(3) $F^{+}(V) \subset \operatorname{cl}\left(\delta-\operatorname{int}\left(F^{+}(\operatorname{cl}(V))\right)\right)$ for any open set $V$,

(4) $\operatorname{int}\left(\delta-\operatorname{cl}\left(F^{-}(V)\right)\right) \subset F^{-}(\mathrm{cl}(V))$ for any open set $V$,

(5) $\operatorname{int}\left(\delta-\operatorname{cl}\left(F^{-}(\operatorname{int}(K))\right)\right) \subset F^{-}(K)$ for any closed set $K$,

(6) $\delta-\operatorname{scl}\left(F^{-}(\operatorname{int}(K))\right) \subset F^{-}(K)$ for any closed set $K$,

(7) $\delta-\operatorname{scl}\left(F^{-}(\operatorname{int}(\operatorname{cl}(B)))\right) \subset F^{-}(\operatorname{cl}(B))$ for any subset $B$,

(8) $F^{+}(\operatorname{int}(B)) \subset \delta$-sint $\left(F^{+}(\operatorname{cl}(\operatorname{int}(B)))\right)$ for any subset $B$,

(9) $F^{+}(V) \subset \delta$-sint $\left(F^{+}(\mathrm{cl}(V))\right)$ for any open set $V$,

(10) $\delta$ - $\operatorname{scl}\left(F^{-}(V)\right) \subset F^{-}(\operatorname{cl}(V))$ for any open set $V$.

Proof. (1) $\Leftrightarrow(2)$ : Obvious.

(1) $\Rightarrow$ (3): Let $V$ be any open set and $x \in F^{+}(V)$. Then $F(x) \subset V$. By (1), there exists a $\delta$-semiopen set $U$ containing $x$ such that $U \subset F^{+}(\operatorname{cl}(V))$. Since $U$ is $\delta$ semiopen, we have $x \in U \subset \operatorname{cl}(\delta$-int $(U)) \subset \operatorname{cl}\left(\delta\right.$-int $\left.\left(F^{+}(\operatorname{cl}(V))\right)\right)$.

(3) $\Rightarrow(4)$ : Let $V$ be any open set. Then we get

$$
\begin{aligned}
X \backslash F^{-}(\operatorname{cl}(V))=F^{+}(Y \backslash \operatorname{cl}(V)) & \subset \operatorname{cl}\left(\delta-\operatorname{int}\left(F^{+}(\operatorname{cl}(Y \backslash \operatorname{cl}(V)))\right)\right) \\
=\operatorname{cl}\left(\delta-\operatorname{int}\left(F^{+}(Y \backslash\right.\right. & \backslash \operatorname{int}(\operatorname{cl}(V))))) \subset \operatorname{cl}\left(\delta-\operatorname{int}\left(F^{+}(Y \backslash V)\right)\right) \\
& =\operatorname{cl}\left(\delta-\operatorname{int}\left(X \backslash F^{-}(V)\right)\right)=X \backslash \operatorname{int}\left(\delta-\operatorname{cl}\left(F^{-}(V)\right)\right) .
\end{aligned}
$$

Hence, we obtain $\operatorname{int}\left(\delta-\operatorname{cl}\left(F^{-}(V)\right)\right) \subset F^{-}(\operatorname{cl}(V))$.

$(4) \Rightarrow(5)$ : Let $K$ be any closed set. Since $\operatorname{int}(K)$ is open, $\operatorname{int}\left(\delta-\operatorname{cl}\left(F^{-}(\operatorname{int}(K))\right)\right) \subset$ $F^{-}(\operatorname{cl}(\operatorname{int}(K))) \subset F^{-}(K)$.

$(5) \Rightarrow(6)$ : Let $K$ be any closed set. Then we have $\operatorname{int}\left(\delta-\operatorname{cl}\left(F^{-}(\operatorname{int}(K))\right)\right) \subset F^{-}(K)$ and $F^{-}(\operatorname{int}(K)) \subset F^{-}(K)$. Since the equality $\delta$-scl $\left(F^{-}(\operatorname{int}(K))\right)=F^{-}(\operatorname{int}(K)) \cup$ $\operatorname{int}\left(\delta\right.$-cl $\left.\left(F^{-}(\operatorname{int}(K))\right)\right)$ holds, we obtain $\delta$-scl $\left(F^{-}(\operatorname{int}(K))\right) \subset F^{-}(K)$.

$(6) \Rightarrow(7)$ : Let $B$ be any subset of $Y$, then $\operatorname{cl}(B)$ is closed in $Y$. Thus we obtain $\delta-\operatorname{scl}\left(F^{-}(\operatorname{int}(\operatorname{cl}(B)))\right) \subset F^{-}(\operatorname{cl}(B))$.

(7) $\Rightarrow(8)$ : Let $B$ be any subset of $Y$. Then, we obtain $F^{+}(\operatorname{int}(B))=X \backslash F^{-}(\operatorname{cl}(Y \backslash$ $B)) \subset X \backslash \delta-\operatorname{scl}\left(F^{-}(\operatorname{int}(\operatorname{cl}(Y \backslash B)))\right)=\delta-\operatorname{sint}\left(F^{+}(\operatorname{cl}(\operatorname{int}(B)))\right)$.

$(8) \Rightarrow(9)$ : Obvious.

$(9) \Rightarrow(10)$ : Let $V$ be any open set. By (9), we get

$$
\begin{aligned}
\delta-\operatorname{scl}\left(F^{-}(V)\right) \subset \delta-\operatorname{scl}\left(F^{-}(\operatorname{int}(\operatorname{cl}(V)))\right)= & \delta-\operatorname{scl}\left(X \backslash F^{+}(Y \backslash \operatorname{int}(\operatorname{cl}(V)))\right) \\
=X \backslash \delta-\operatorname{sint}\left(F^{+}(Y \backslash \operatorname{int}(\operatorname{cl}(V)))\right)= & X \backslash \delta-\operatorname{sint}\left(F^{+}(\operatorname{cl}(Y \backslash \operatorname{cl}(V)))\right) \\
& \subset X \backslash F^{+}(Y \backslash \operatorname{cl}(V))=F^{-}(\operatorname{cl}(V)) .
\end{aligned}
$$

Hence, $\delta$-scl $\left(F^{-}(V)\right) \subset F^{-}(\operatorname{cl}(V))$.

$(10) \Rightarrow(1)$ : Let $V$ be any open set and $F(x) \subset V$. Set $U=\delta-\operatorname{sint}\left(F^{+}(\operatorname{cl}(V))\right)$. Then we have $U=\delta$-sint $\left(F^{+}(\operatorname{cl}(V))\right)=X \backslash \delta-\operatorname{scl}\left(F^{-}(Y \backslash \operatorname{cl}(V))\right) \supset X \backslash F^{-}(\operatorname{cl}(Y \backslash$ $\operatorname{cl}(V)))=F^{+}(\operatorname{int}(\operatorname{cl}(V))) \supset F^{+}(V)$ and $x \in F^{+}(V)$. Hence, $U \in \delta S O(X, x)$ and $F(U) \subset \operatorname{cl}(V)$. This shows that $F$ is upper weakly $\delta$-semicontinuous. 
Remark 1. For a multifunction $F: X \rightarrow Y$, the following implications hold:

upper weakly $\delta$-semicontinuous

$\Uparrow$

upper almost $\delta$-semicontinuous

$\Uparrow$

upper $\delta$-semicontinuous

$\Downarrow$

upper quasi-continuous

The following examples show that these implications are not reversible.

Example 1. Let $X=\{a, b, c\}$. Let $\tau$ be a topology on $X$ given by the relation $\tau=$ $\{\varnothing, X,\{a\},\{c\},\{a, c\},\{b, c\}\}$. Define the multifunction $F: X \rightarrow X$ by $F(a)=\{a\}$, $F(b)=\{b\}$ and $F(c)=\{c\}$. Then $F$ is upper almost $\delta$-semicontinuous but not upper $\delta$-semicontinuous.

Example 2. Let $X=\{a, b, c, d\}$. Let $\tau$ be a topology on $X$ given by the relation $\tau=\{\varnothing, X,\{a\},\{c\},\{a, b\},\{a, c\},\{a, b, c\},\{a, c, d\}\}$. Define the multifunction $F: X \rightarrow X$ by $F(a)=\{a, b\}, F(b)=\{d\}, F(c)=\{a, c, d\}$ and $F(d)=\{a, c, d\}$. Then $F$ is upper weakly $\delta$-semicontinuous but not upper almost $\delta$-semicontinuous.

We refer the reader to [3] for an example concerning the remaining implication.

Theorem 4. Let $F: X \rightarrow Y$ be a multifunction. Then the following statements are equivalent:

(1) $F$ is lower weakly $\delta$-semicontinuous multifunction,

(2) for each $x \in X$ and each open set $V$ such that $F(x) \cap V \neq \varnothing$, there exists $a \delta$-semiopen set $U$ containing $x$ such that if $y \in U$, then $F(y) \cap \operatorname{cl}(V) \neq \varnothing$,

(3) $F^{-}(V) \subset \operatorname{cl}\left(\delta-\operatorname{int}\left(F^{-}(\operatorname{cl}(V))\right)\right)$ for any open set $V$,

(4) $\operatorname{int}\left(\delta-\operatorname{cl}\left(F^{+}(V)\right)\right) \subset F^{+}(\operatorname{cl}(V))$ for any open set $V$,

(5) $\operatorname{int}\left(\delta-\operatorname{cl}\left(F^{+}(\operatorname{int}(K))\right)\right) \subset F^{+}(K)$ for any closed set $K$,

(6) $\delta-\operatorname{scl}\left(F^{+}(\operatorname{int}(K))\right) \subset F^{+}(K)$ for any closed set $K$,

(7) $\delta-\operatorname{scl}\left(F^{+}(\operatorname{int}(\operatorname{cl}(B)))\right) \subset F^{+}(\operatorname{cl}(B))$ for any subset $B$,

(8) $F^{-}(\operatorname{int}(B)) \subset \delta$-sint $\left(F^{-}(\operatorname{cl}(\operatorname{int}(B)))\right)$ for any subset $B$,

(9) $F^{-}(V) \subset \delta$-sint $\left(F^{-}(\mathrm{cl}(V))\right)$ for any open set $V$,

(10) $\delta$-scl $\left(F^{+}(V)\right) \subset F^{+}(\operatorname{cl}(V))$ for any open set $V$.

Proof. The proof is similar to that of the previous theorem.

Theorem 5. Let $F: X \rightarrow \prod_{i \in I} X_{i}$ be a multifunction from a topological space $X$ to the product space $\prod_{i \in I} X_{i}$ and let $p_{i}: \prod_{i \in I} X_{i} \rightarrow X_{i}$ be the projection for each $i \in I$. If $F$ is upper (lower) weakly $\delta$-semicontinuous multifunction, then $p_{i} \circ F$ is upper (resp., lower) weakly $\delta$-semicontinuous multifunction for each $i \in I$. 
Proof. We shall prove this only for the upper continuity.

Let $V_{i_{0}}$ be an open set in $X_{i_{0}}$. We have $\left(p_{i_{0}} \circ F\right)^{+}\left(V_{i_{0}}\right)=F^{+}\left(p_{i_{0}}^{+}\left(V_{i_{0}}\right)\right)=$ $F^{+}\left(V_{i_{0}} \times \prod_{i \in I} X_{i}\right)$. Take $x \in\left(p_{i_{0}} \circ F\right)^{+}\left(V_{i_{0}}\right)$. Since $F$ is upper weakly $\delta$-semicontinuous and $V_{i_{0}} \times \prod_{i \in I} X_{i}$ is an open set, there exists a $\delta$-semiopen set $U$ containing $x$ such that $U \subset F^{+}\left(\operatorname{cl}\left(V_{i_{0}} \times \prod_{i \in I} X_{i}\right)\right)$. Since $F^{+}\left(\operatorname{cl}\left(V_{i_{0}} \times \prod_{i \in I} X_{i}\right)\right)=$ $F^{+}\left(\operatorname{cl}\left(V_{i_{0}}\right) \times \prod_{i \in I} X_{i}\right)=\left(p_{i_{0}} \circ F\right)^{+}\left(\operatorname{cl}\left(V_{i_{0}}\right)\right)$, then $p_{i_{0}} \circ F$ is upper weakly $\delta$-semicontinuous multifunction.

Theorem 6. Let $F: X \rightarrow Y$ be a multifunction and let $S$ be a $\delta$-open set in $X$. If $F$ is a lower (upper) weakly $\delta$-semicontinuous, then the restriction multifunction $\left.F\right|_{S}: S \rightarrow Y$ is a lower (resp., upper) weakly $\delta$-semicontinuous.

Proof. Suppose that $V$ is an open set in $Y$. Let $x \in S$ and let $x \in\left(\left.F\right|_{S}\right)^{-}(V)$. Since $F$ is lower weakly $\delta$-semicontinuous multifunction, it follows that there exists a $\delta$-semiopen set $G$ such that $x \in G \subset F^{-}(\operatorname{cl}(V))$. We obtain $x \in G \cap S \in \delta S O(S)$ and $G \cap S \subset\left(\left.F\right|_{S}\right)^{-}(\operatorname{cl}(V))$. Thus, we show that the restriction multifunction $\left.F\right|_{S}$ is a lower weakly $\delta$-semicontinuous.

The proof of the upper weakly $\delta$-semicontinuity of $\left.F\right|_{S}$ is similar to that given above.

Theorem 7. Let $F: X \rightarrow Y$ be a multifunction. If the graph multifunction of $F$ is upper weakly $\delta$-semicontinuous, then $F$ is upper weakly $\delta$-semicontinuous.

Proof. Suppose that $G_{F}: X \rightarrow X \times Y$ is upper weakly $\delta$-semicontinuous. Let $x \in$ $X$ and $V$ be any open set of $Y$ containing $F(x)$. Since $X \times V$ is open in $X \times Y$ and $G_{F}(x) \subset X \times V$, there exists $U \in \delta S O(X, x)$ such that $G_{F}(U) \subset \operatorname{cl}(X \times V)=X \times$ $\operatorname{cl}(V)$. By Lemma 1, we have $U \subset G_{F}^{+}(X \times \operatorname{cl}(V))=F^{+}(\operatorname{cl}(V))$ and $F(U) \subset \operatorname{cl}(V)$. This shows that $F$ is upper weakly $\delta$-semicontinuous.

Theorem 8. A multifunction $F: X \rightarrow Y$ is lower weakly $\delta$-semicontinuous provided that $G_{F}: X \rightarrow X \times Y$ is lower weakly $\delta$-semicontinuous.

Proof. Suppose that $G_{F}$ is lower weakly $\delta$-semicontinuous. Let $x \in X$ and $V$ be any open set of $Y$ such that $x \in F^{-}(V)$. Then $X \times V$ is open in $X \times Y$ and $G_{F}(x) \cap(X \times V)=(\{x\} \times F(x)) \cap(X \times V)=\{x\} \times(F(x) \cap V) \neq \varnothing$. Since $G_{F}$ is lower weakly $\delta$-semicontinuous, there exists a $\delta$-semiopen set $U$ containing $x$ such that $U \subset G_{F}^{-}(\operatorname{cl}(X \times V))$. Since $G_{F}^{-}(\operatorname{cl}(X \times V))=G_{F}^{-}(X \times \operatorname{cl}(V))$, by Lemma 1, we have $U \subset F^{-}(\operatorname{cl}(V))$. This shows that $F$ is lower weakly $\delta$-semicontinuous.

Theorem 9. Suppose that $F_{1}: X \rightarrow Y, F_{2}: X \rightarrow Z$ are multifunctions. Let $F_{1} \times$ $F_{2}: X \rightarrow Y \times Z$ be a multifunction which is defined by $\left(F_{1} \times F_{2}\right)(x)=F_{1}(x) \times F_{2}(x)$ for each $x \in X$. If $F_{1} \times F_{2}$ is upper (lower) weakly $\delta$-semicontinuous multifunction, then $F_{1}$ and $F_{2}$ are upper (resp., lower) weakly $\delta$-semicontinuous multifunctions.

Proof. Let $x \in X$ and let $K \subset Y, H \subset Z$ be open sets such that $x \in F_{1}^{+}(K)$ and $x \in F_{2}^{+}(H)$. Then we obtain that $F_{1}(x) \subset K$ and $F_{2}(x) \subset H$ and so $F_{1}(x) \times$ 
$F_{2}(x)=\left(F_{1} \times F_{2}\right)(x) \subset K \times H$. We have $x \in\left(F_{1} \times F_{2}\right)^{+}(K \times H)$. Since $F_{1} \times$ $F_{2}$ is upper weakly $\delta$-semicontinuous multifunction, it follows that there exists a $\delta$ semiopen set $U$ containing $x$ such that $U \subset\left(F_{1} \times F_{2}\right)^{+}(\operatorname{cl}(K \times H))$. We obtain that $U \subset F_{1}^{+}(\operatorname{cl}(K))$ and $U \subset F_{2}^{+}(\operatorname{cl}(H))$. Thus, we obtain that $F_{1}$ and $F_{2}$ are upper weakly $\delta$-semicontinuous multifunctions.

The proof of the lower weakly $\gamma$-continuity of $F_{1}$ and $F_{2}$ is similar to that presented above.

Definition 6. Let $F: X \rightarrow Y$ be a multifunction. The multigraph $G(F)$ is said to be $\delta_{s}$-graph in $X \times Y$ if for each $(x, y) \notin G(F)$, there exist $\delta$-semiopen set $U$ and open set $V$ containing $x$ and $y$, respectively, such that $(U \times V) \cap G(F)=\varnothing$.

Definition 7. A subset $A$ of a topological space $X$ is said to be $\alpha$-paracompact [17] if every cover of $A$ by open sets of $X$ is refined by a cover of $A$ which consists of open sets of $X$ and is locally finite in $X$.

Furthermore, a multifunction $F:(X, \tau) \rightarrow(Y, \sigma)$ is called punctually $\alpha$-paracompact [13] if $F(x)$ is $\alpha$-paracompact for each point $x \in X$.

Theorem 10. Let $F:(X, \tau) \rightarrow(Y, \sigma)$ be an upper weakly $\delta$-semicontinuous and punctually $\alpha$-paracompact multifunction into a Hausdorff space $(Y, \sigma)$. Then the multigraph $G(F)$ of $F$ is a $\delta_{s}$-graph in $X \times Y$.

Proof. Suppose that $\left(x_{0}, y_{0}\right) \notin G(F)$. Then $y_{0} \notin F\left(x_{0}\right)$. Since $(Y, \sigma)$ is a Hausdorff space, for each $y \in F\left(x_{0}\right)$, there exist open sets $V(y)$ and $W(y)$ containing $y$ and $y_{0}$, respectively, such that $V(y) \cap W(y)=\varnothing$. The family $\{V(y): y \in$ $\left.F\left(x_{0}\right)\right\}$ is an open cover of $F\left(x_{0}\right)$ which is $\alpha$-paracompact. Thus, it has a locally finite open refinement $\Phi=\left\{U_{\beta}: \beta \in I\right\}$ which covers $F\left(x_{0}\right)$. Let $W_{0}$ be an open neighborhood of $y_{0}$ such that $W_{0}$ intersects only finitely many members $U_{\beta_{1}}, U_{\beta_{2}}$, $\ldots, U_{\beta_{n}}$ of $\Phi$. Choose $y_{1}, y_{2}, \ldots, y_{n}$ in $F\left(x_{0}\right)$ such that $U_{\beta_{i}} \subset V\left(y_{i}\right)$ for each $i=1,2, \ldots, n$ and set $W=W_{0} \cap\left(\cap_{i=1}^{n} W\left(y_{i}\right)\right)$. Then $W$ is an open neighborhood of $y_{0}$ with $W \cap\left(\cup_{\beta \in I} U_{\beta}\right)=\varnothing$, which implies that $W \cap \operatorname{cl}\left(\cup_{\beta \in I} U_{\beta}\right)=\varnothing$. By the upper weakly $\delta$-semicontinuity of $F$, there exists $U \in \delta S O\left(X, x_{0}\right)$ such that $F(U) \subset \operatorname{cl}\left(\cup_{\beta \in I} U_{\beta}\right)$. It follows that $(U \times W) \cap G(F)=\varnothing$. Hence, the graph $G(F)$ is a $\delta_{s}$-graph in $X \times Y$.

Definition 8. A space $X$ is said to be semi-connected [11] if it cannot be expressed as the union of two non-empty disjoint semiopen sets.

Lemma 3 ([8]). For a space $X$, the following properties are equivalent:

(1) $X$ is semi-connected,

(2) $X$ cannot be expressed as the union of two nonempty disjoint $\delta$-semiopen sets.

Theorem 11. Let $F$ be a multifunction from a semi-connected topological space $X$ onto a topological space $Y$ such that $F$ is point connected. If $F$ is upper almost $\delta$-semicontinuous multifunction, then $Y$ is a connected space. 
Proof. Let $F: X \rightarrow Y$ be a upper almost $\delta$-semicontinuous multifunction from a semi-connected topological space $X$ onto a topological space $Y$. Suppose that $Y$ is not connected and let $Y=H \cup K$ be a partition of $Y$. Then both $H$ and $K$ are open and closed subsets of $Y$. Since $F$ is upper almost $\delta$-semicontinuous multifunction, $F^{+}(H)$ and $F^{+}(K)$ are $\delta$-semiopen subsets of $X$. In view of the fact that $F^{+}(H)$, $F^{+}(K)$ are disjoint and $F$ is point connected, $X=F^{+}(H) \cup F^{+}(K)$ is a partition of $X$. This is contrary to the semi-connectedness of $X$. Hence, it is obtained that $Y$ is a connected space.

Definition 9. A topological space $(X, \tau)$ is said to be semi- $T_{2}[5]$ if, for each distinct points $x, y \in X$, there exist semiopen sets $U$ and $V$ containing $x$ and $y$, respectively, such that $U \cap V=\varnothing$.

Lemma 4 ([8]). For a space $X$, the following properties are equivalent:

(1) $\mathrm{X}$ is semi- $T_{2}$,

(2) for each distinct points $x, y \in X$, there exist $U, V \in \delta S O(X)$ containing $x$ and $y$, respectively, such that $U \cap V=\varnothing$.

Theorem 12. Let $F: X \rightarrow Y$ be an upper almost $\delta$-semicontinuous multifunction and point closed from a topological space $X$ to a normal topological space $Y$ and let $F(x) \cap F(y)=\varnothing$ for each distinct pair $x, y \in X$. Then $X$ is a semi- $T_{2}$.

Proof. Let $x$ and $y$ be any two distinct points in $X$. Then we have $F(x) \cap F(y)=$ $\varnothing$. Since $Y$ is a normal space, it follows that there exists disjoints open sets $U$ and $V$ containing $F(x)$ and $F(y)$, respectively. Thus $F^{+}(\operatorname{int}(\operatorname{cl}(U)))$ and $F^{+}(\operatorname{int}(\operatorname{cl}(V)))$ are disjoint $\delta$-semiopen sets containing $x$ and $y$, respectively. Thus, it is obtained that $X$ is semi- $T_{2}$.

Recall that the $\delta$-semifrontier of a subset $A$ of a space $X$, denoted by $\delta$ - $\operatorname{sfr}(A)$, is defined by $\delta-\operatorname{sfr}(A)=\delta-\operatorname{scl}(A) \cap \delta-\operatorname{scl}(X \backslash A)=\delta-\operatorname{scl}(A) \backslash \delta-\operatorname{sint}(A)$.

Theorem 13. The set all points of $X$ at which a multifunction $F: X \rightarrow Y$ is not upper almost $\delta$-semicontinuous (lower almost $\delta$-semicontinuous) is identical with the union of the $\delta$-semifrontier of the upper (lower) inverse images of regular open sets containing (meeting) $F(x)$.

Proof. Let $x \in X$ at which $F$ is not upper almost $\delta$-semicontinuous. Then there exists a regular open set $V$ of $Y$ containing $F(x)$ such that $U \cap\left(X \backslash F^{+}(V)\right) \neq$ $\varnothing$ for every $U \in \delta S O(X, x)$. Therefore, we have $x \in \delta$-scl $\left(X \backslash F^{+}(V)\right)=X \backslash \delta$ $\operatorname{sint}\left(F^{+}(V)\right)$ and $x \in F^{+}(V)$. Thus, we obtain $x \in \delta$-sfr $\left(F^{+}(V)\right)$.

Conversely, suppose that $V$ is a regular open set of $Y$ containing $F(x)$ such that $x \in \delta$-sfr $\left(F^{+}(V)\right)$. If $F$ is upper almost $\delta$-semicontinuous at $x$, then there exists $U \in$ $\delta S O(X, x)$ such that $U \subset F^{+}(V)$; hence $x \in \delta$-sint $\left(F^{+}(V)\right)$. This is a contradiction and hence $F$ is not upper almost $\delta$-semicontinuous at $x$.

The case of the lower almost $\delta$-semicontinuity can be considered similarly. 


\section{RELATION BETWEEN TYPES OF $\delta$-SEMICONTINUITY}

In this section, the relationships among $\delta$-semicontinuous multifunctions, almost $\delta$-semicontinuous multifunctions and weakly $\delta$-semicontinuous multifunctions are investigated.

Definition 10. A multifunction $F: X \rightarrow Y$ is said to be almost $\delta$-semiopen if $F(U) \subset \operatorname{int}(\operatorname{cl}(F(U)))$ for every $\delta$-semiopen set $U$ in $X$.

Theorem 14. If a multifunction $F: X \rightarrow Y$ is upper weakly $\delta$-semicontinuous and almost $\delta$-semiopen, then $F$ is upper almost $\delta$-semicontinuous.

Proof. Let $V$ be an arbitrary open set containing $F(x)$. There exists a $\delta$-semiopen set $U$ containing $x$ such that $F(U) \subset \operatorname{cl}(V)$. Since $F$ is almost $\delta$-semiopen, we have $F(U) \subset \operatorname{int}(\operatorname{cl}(F(U))) \subset \operatorname{int}(\operatorname{cl}(V))$. It then follows that $F$ is upper almost $\delta$-semicontinuous.

Theorem 15. Let $F: X \rightarrow Y$ be a multifunction such that $F(x)$ is an open set of $Y$ for each $x \in X$. Then the following assertions are equivalent:

(1) $F$ is lower $\delta$-semicontinuous,

(2) $F$ is lower almost $\delta$-semicontinuous,

(3) $F$ is lower weakly $\delta$-semicontinuous.

Proof. (1) $\Rightarrow(2),(2) \Rightarrow(3)$ : Obvious.

(3) $\Rightarrow(1)$ : Let $x \in X$ and $V$ be an open set of $Y$ such that $F(x) \cap V \neq \varnothing$. Then there exists a $\delta$-semiopen set $U$ containing $x$ such that $F(u) \cap \operatorname{cl}(V) \neq \varnothing$ for each $u \in U$. Since $F(u)$ is open, $F(u) \cap V \neq \varnothing$ for each $u \in U$ and hence $F$ is lower $\delta$-semicontinuous.

Theorem 16. If $F: X \rightarrow Y$ is lower weakly $\delta$-semicontinuous and there exists an open basis $\Psi=\left\{V_{i}: i \in I\right\}$ of the topology for $Y$ such that $F^{-}\left(\operatorname{cl}\left(V_{i}\right)\right) \subset F^{-}\left(V_{i}\right)$ for every $i \in I$, then $F$ is lower $\delta$-semicontinuous.

Proof. Let $\Psi=\left\{V_{i}: i \in I\right\}$ be an open basis of the topology for $Y$ such that $F^{-}\left(\mathrm{cl}\left(V_{i}\right)\right) \subset F^{-}\left(V_{i}\right)$ for every $i \in I$. For any open set $V$, there exists a subset $\Psi_{0}$ of $\Psi$ such that $V=\cup_{i \in \Psi_{0}} V_{i}$. Therefore, by Theorem 4, we obtain

$$
\begin{aligned}
F^{-}(V)=F^{-}\left(\cup_{i \in \Psi_{0}} V_{i}\right)=\cup_{i \in \Psi_{0}} F^{-}\left(V_{i}\right) \subset \cup_{i \in \Psi_{0}} \delta-\operatorname{sint}\left(F^{-}\left(\operatorname{cl}\left(V_{i}\right)\right)\right) \\
\subset \cup_{i \in \Psi_{0}} \delta-\operatorname{sint}\left(F^{-}\left(V_{i}\right)\right) \subset \delta-\operatorname{sint}\left(\cup_{i \in \Psi_{0}} F^{-}\left(V_{i}\right)\right) \\
=\delta-\operatorname{sint}\left(F^{-}\left(\cup_{i \in \Psi_{0}} V_{i}\right)\right)=\delta-\operatorname{sint}\left(F^{-}(V)\right) .
\end{aligned}
$$

This shows that $F$ is lower $\delta$-semicontinuous.

Theorem 17. If $F: X \rightarrow Y$ is upper weakly $\delta$-semicontinuous and satisfies the condition $F^{+}(\mathrm{cl}(V)) \subset F^{+}(V)$ for every open set $V$ of $Y$, then $F$ is upper $\delta$ semicontinuous. 
Proof. Let $V$ be any open set. Since $F$ upper weakly $\delta$-semicontinuous, we get the inclusion $F^{+}(V) \subset \delta$-sint $\left(F^{+}(\operatorname{cl}(V))\right)$ and hence $F^{+}(V) \subset \delta$-sint $\left(F^{+}(\operatorname{cl}(V))\right) \subset \delta$ $\operatorname{sint}\left(F^{+}(V)\right)$. Thus, $F^{+}(V)$ is $\delta$-semiopen and $F$ is upper $\delta$-semicontinuous.

Theorem 18. Let $F: X \rightarrow Y$ be a multifunction such that $F(x)$ is closed in $Y$ for each $x \in X$ and $Y$ is normal. Then the following assertions are equivalent:

(1) $F$ is upper weakly $\delta$-semicontinuous,

(2) $F$ is upper almost $\delta$-semicontinuous,

(3) $F$ is upper $\delta$-semicontinuous.

Proof. (3) $\Rightarrow(2),(2) \Rightarrow(1)$ : Obvious.

$(1) \Rightarrow(3)$ : Suppose that $F$ is upper weakly $\delta$-semicontinuous. Let $x \in X$ and $G$ be an open set containing $F(x)$. Since $F(x)$ is closed in $Y$, by the normality of $Y$, there exists an open set $V$ such that $F(x) \subset V \subset \operatorname{cl}(V) \subset G$. Since $F$ is upper weakly $\delta$-semicontinuous, there exists a $\delta$-semiopen set $U$ containing $x$ such that $F(U) \subset \operatorname{cl}(V) \subset G$. This shows that $F$ is upper $\delta$-semicontinuous.

\section{REFERENCES}

[1] M. E. Abd El-Monsef, S. N. El-Deeb, and R. A. Mahmoud, “ $\beta$-open sets and $\beta$-continuous mapping," Bull. Fac. Sci. Assiut Univ. A, vol. 12, no. 1, pp. 77-90, 1983.

[2] S. G. Crossley and S. K. Hildebrand, "Semi-closure," Texas J. Sci., vol. 22, pp. 99-112, 1971.

[3] E. Ekici, "On lower and upper $\delta$-semicontinuous multifunctions," Int. J. Pure Appl. Math., vol. 21, no. 1, pp. 9-17, 2005.

[4] N. Levine, "Semi-open sets and semi-continuity in topological spaces," Amer. Math. Monthly, vol. 70, pp. 36-41, 1963.

[5] S. N. Maheshwari and R. Prasad, "Some new separation axioms," Ann. Soc. Sci. Bruxelles Sér. I, vol. 89, no. 3, pp. 395-402, 1975.

[6] A. S. Mashhour, M. E. Abd El-Monsef, and S. N. El-Deep, "On precontinuous and weak precontinuous mappings," Proc. Math. Phys. Soc. Egypt, no. 53, pp. 47-53 (1983), 1982.

[7] T. Noiri, "Almost quasi-continuous functions," Bull. Inst. Math. Acad. Sinica, vol. 18, no. 4, pp. 321-332, 1990.

[8] T. Noiri, "Remarks on $\delta$-semi-open sets and $\delta$-preopen sets," Demonstratio Math., vol. 36, no. 4, pp. 1007-1020, 2003.

[9] T. Noiri and V. Popa, "Almost weakly continuous multifunctions," Demonstratio Math., vol. 26, no. 2, pp. 363-380, 1993.

[10] J. H. Park, B. Y. Lee, and M. J. Son, “On $\delta$-semiopen sets in topological space," J. Indian Acad. Math., vol. 19, no. 1, pp. 59-67, 1997.

[11] V. Pipitone and G. Russo, "Spazi semiconnessi e spazi semiaperti," Rend. Circ. Mat. Palermo (2), vol. 24, no. 3, pp. 273-285, 1975.

[12] V. Popa and T. Noiri, "On upper and lower $\alpha$-continuous multifunctions," Math. Slovaca, vol. 43, no. 4, pp. 477-491, 1993.

[13] V. Popa and T. Noiri, "On upper and lower $\alpha$-continuous multifunctions," Math. Slovaca, vol. 43, no. 4, pp. 477-491, 1993.

[14] R. E. Smithson, "Almost and weak continuity for multifunctions," Bull. Calcutta Math. Soc., vol. 70, no. 6, pp. 383-390, 1978.

[15] M. H. Stone, "Applications of the theory of Boolean rings to general topology," Trans. Amer. Math. Soc., vol. 41, no. 3, pp. 375-481, 1937. 
[16] N. V. Veličko, " $H$-closed topological spaces," Mat. Sb. (N.S.), vol. 70 (112), pp. 98-112, 1966, English transl. in Amer. Math. Soc. Transl., II. Ser., vol. 78, pp. 103-118, 1968.

[17] J. D. Wine, "Locally paracompact spaces," Glasnik Mat. Ser. III, vol. 10(30), no. 2, pp. 351-357, 1975.

\section{Author's address}

\section{Erdal Ekici}

Department of Mathematics, Canakkale Onsekiz Mart University, Terzioglu Campus, 17020 Canakkale, Turkey

E-mail address: eekiciecomu.edu.tr 